\title{
Transcatheter aortic valve implantation in high-risk patients with severe aortic stenosis reduces 1-year mortality compared to standard therapy
}

\author{
Monica Solbiati · Marta Prado
}

Received: 3 January 2011 / Accepted: 17 February 2011/Published online: 5 March 2011

(c) SIMI 2011

\section{Background}

Aortic stenosis is one of the most common valvular disorders in older adults, with an incidence of $8 \%$ at age 85 [2]. In adults with severe symptomatic aortic stenosis, 2-year mortality is approximately $50 \%$ [2] and open surgical aortic valve replacement (SAVR) is the only treatment that has been shown to improve symptoms, functional status and survival [3]. However, nearly $30 \%$ of patients with severe and symptomatic aortic valve stenosis are not suitable candidates for surgical treatment because of comorbidities and high surgical risk [4].

A recent systematic review of 84 case-series, which included 2,375 patients older than 80 and at high surgical risk undergoing transcatheter aortic valve implantation (TAVI), showed a successful implantation in $94 \%$ of patients and a 30-day survival of $89 \%$ [5].

\section{Summary}

The PARTNER Trial compared transcatheter aortic valve implantation (TAVI) with standard therapy (including balloon aortic valvuloplasty) in high-risk patients with severe aortic stenosis [1]. 358 patients (mean age 83 years)

Monica Solbiati and Marta Prado on behalf of GrAM.

M. Solbiati $(\bowtie) \cdot$ M. Prado

Medicina Interna II, Dipartimento di Scienze Cliniche, Ospedale

L. Sacco, Università degli Studi di Milano, Via GB grassi 74,

20157 Milan, Italy

e-mail: monica.solbiati@gmail.com were randomized to TAVI (Edwards SAPIEN heart-valve system) or to standard therapy. The primary end point was the rate of death from any cause. At 1 year, it was $30.7 \%$ with TAVI, as compared to $50.7 \%$ in the standard therapy group (hazard ratio with TAVI, $0.55 ; 95 \%$ confidence interval [CI], 0.40-0.74; $P<0.001$ ) with a number needed to treat of five. Among survivors at 1 year, the rate of cardiac symptoms (New York Heart Association class III or IV) was also lower among TAVI patients (25.2 vs. $58.0 \%, P<0.001)$. However, at 30 days, TAVI, was associated with a higher incidence of major strokes (5.0 vs. $1.1 \%, P=0.06$ ), major vascular complications (16.2 vs. $1.1 \%, P<0.001)$ and major bleeding (16.8 vs. $3.9 \%$, $P<0.001)$. The procedure was performed under general anaesthesia; adjunctive therapy included heparin during the procedure and dual antiplatelet therapy for 6 months.

\section{Strengths of the study}

- It addresses an important issue in the daily clinical practice: patients with severe aortic stenosis not suitable for surgical aortic valve replacement;

- a strong end point (mortality at 1 year) was considered;

- it is the first randomized controlled trial about TAVI (good internal validity);

- it included a cohort of patients similar to that seen in everyday clinical practice (good external validity).

\section{Weakness of the study}

- TAVI is a relatively new procedure, and we cannot assume that the same results in terms of safety and effectiveness as those reported in the study will be 
obtained by relatively inexperienced interventional cardiologists;

- the trial is sponsored by the aortic valve manufacturer, which designed the trial, funded the studies, and was involved in the data collection and monitoring.

\section{Question marks}

- It would be interesting to know which medical therapy was considered as standard in the "standard therapy" group;

- it is not clear how many "standard therapy" patients underwent balloon aortic valvuloplasty (discordance between text and tables), and since there are no conclusive results about this procedure, we do not know how this could have affected the results.

\section{Clinical bottom line}

TAVI, performed by physicians with strong expertise in this technique, should be considered for patients with aortic stenosis who are not suitable candidates for surgery. The higher short-term risk must be balanced with the better long term prognosis (1 year), and the specific patients willing to choose the best therapeutic strategy. Additional randomized trials are needed to compare TAVI with aortic valve replacement among high-risk patients affected by aortic stenosis for whom surgery is still a viable option.

Conflict of interest None.

\section{References}

1. Leon MB, Smith CR, Mack M et al (2010) Transcatheter aorticvalve implantation for aortic stenosis in patients who cannot undergo surgery. N Engl J Med 363:1597-1607

2. Ambler G, Omar RZ, Royston P et al (2005) Generic, simple risk stratification model for heart valve surgery. Circulation 112: 224-231

3. Bonow RO, Carabello BA, Kanu C et al (2006) American College of Cardiology/American Heart Association Task Force on Practice Guidelines. ACC/AHA 2006 guidelines for the management of patients with valvular heart disease: a report of the American College of Cardiology/American Heart Association Task Force on Practice Guidelines. Circulation 114:e84-e231

4. Iung B, Gohlke-Barwolf C, Tornos P, Working group on valvular heart disease et al (2002) Recommendations on the management of the asymptomatic patient with valvular heart disease. Eur Heart J 23:1252-1266

5. Coeytaux RR, Williams JW Jr, Gray RN et al (2010) Percutaneous heart valve replacement for aortic stenosis: state of the evidence. Ann Intern Med 153:314-324 